\title{
Fuerza muscular máxima en atletas con discapacidad intelectual
}

\author{
Carlos Alberto Morales Salas ${ }^{a}$, Kalia lavaut Sánchez ${ }^{b}$ y Rosa María lam ${ }^{c}$ \\ aMsC Control Médico del Entrenamiento Deportivo. Centro Provincial de Medicina del Deporte. Ciudad de la Habana. Cuba. \\ 'Especialista de Primer Grado en Genética Clínica. Centro Provincial de Genética. Ciudad de la Habana. Cuba. \\ 'Especialista de Primer Grado en Bioestadística. Instituto de Hematología. Ciudad de la Habana. Cuba.
}

\section{RESUMEN}

La actividad deportiva requiere la ejecución de movimientos armónicos y bien estructurados, en los que el desarrollo y la maduración del sistema nervioso central y el sistema musculoesquelético desempeñan un papel fundamental. Los atletas con un grado de discapacidad intelectual que no les impide la realización de actividades deportivas, a pesar de ser muy competitivos, tienen rendimientos influidos por el grado de daño neurológico y por su repercusión en la actividad muscular. Se estudiaron 30 atletas masculinos que participaron en los $V$ Juegos Nacionales de Olimpiadas Especiales, en noviembre del 2006, en levantamiento de pesas, que presentaron diferentes causas de discapacidad intelectual (genéticas, 7; prenatales ambientales, 4; perinatales, 7; multifactoriales, 3; desconocidas, 9), para comparar el resultado deportivo y su relación con las variables peso, edad y grado de discapacidad. Para el análisis estadístico se utilizó como medida de tendencia central la mediana, la desviación típica como medida de dispersión de los datos y el test de Mann-Whitney para la comparación de las muestras, tomando como diferencia significativa $p<0,05$. Los resultados mostraron que no hubo correlación entre rendimiento deportivo y las variables peso-edad. Se encontró diferencia significativa $(p=0,002)$ entre el rendimiento deportivo de los atletas con discapacidad moderada y los atletas con discapacidad ligera, y fue más elevada en estos últimos, en las tres modalidades en que se compitió. Las diferencias encontradas, aunque similares a estudios anteriores, pueden estar influidas por las características de la muestra.

PALABRAS CLAVE: Discapacidad intelectual. Rendimiento. Síndrome de Down. Grado de discapacidad. Fuerza muscular.

\begin{abstract}
The sports activity requires of the harmonic and fine movement, and development and maturity of central nervous system and muscle-skeletal system is very important. Children with Down syndrome seen to love sports and can be very competitive, the performance were influenced for the neurological damage and reflect in the muscular activity. In the Down' syndrome occur because the chromosomal abnormality results in a defect in production of normal collagen in the generalize laxity ligamentous and decrease muscle tone. Thirty men athletes was participated in $\mathrm{V} \mathrm{Na-}$ tional Game, 2006 in weightlifting with different causes for discapacity (genetic, 7; environmental, 4; multifactors, 3; unknown, 9; perinatal, 7) were select for determinate the relation among anthropologic measure (weight, age), and degree of discapacity and sportive result. Data are presented as mean \pm SD. The significance of differences was evaluated by Mann-Whitney test. $\mathrm{P}<0,05$ was considered significant. In this study we found that Down' syndrome athletes present lowest sportive result that the others intellectual discapacity with same degree for discapacity. Not found relation between anthropologic measure and performance.
\end{abstract}

KEYWORDS: Intellectual discapacity. Performance. Down syndrome. Discapacity degree. Strength. 


\section{INTRODUCCIÓN}

La actividad deportiva requiere la ejecución de movimientos armónicos y bien estructurados, donde la integración de diferentes sistemas -entre ellos el sistema nervioso central (SNC) y el sistema musculoesquelético- desempeña un papel fundamental para el éxito del gesto deportivo.

La representación motora se basa en un conjunto de movimientos bien aprendidos surgidos de un aprendizaje previo, la acción se automatiza y se almacena en la memoria para su uso posterior $^{1}$. Se sugiere que la corteza parietal y frontal son los sustratos neuronales donde se almacenan las representaciones internas de las acciones motoras. Las neuronas en la corteza deben codificar los aspectos dinámicos del movimiento, dirección y fuerza.

Las representaciones motoras pueden mostrar dos modelos de activación cerebral, que pueden actuar de manera aislada o simultánea: un modelo que activa la corteza parietal y frontal que transfiere las informaciones sensoriomotoras y los movimientos automatizados, y otro modelo que activa la corteza parietal, prefrontal y frontal, que se utiliza para la planificación y la preparación y para el aprendizaje motor ${ }^{2}$. Las vías del sistema visual de Goodale podrían desempeñar un papel determinante en la transformación de las representaciones motoras en movimiento ${ }^{3}$.

En atletas con discapacidad intelectual (DI) el desempeño deportivo está influido por sus alteraciones, características morfofuncionales propias de su patología y de la gravedad del dańo neuromuscular. Definimos la DI como el estado caracterizado por limitaciones significativas en el funcionamiento intelectual y la conducta adaptativa que se manifiesta en actividades prácticas, sociales y conceptuales, con inicio antes de los 18 años ${ }^{4}$. Clínicamente, la DI se caracteriza por dismorfia típica, hiperlaxitud articular e hipotonía muscular, presente estas últimas en el $85 \%$ de los casos.

La DI presenta diferentes etiologías, y la causa genética más frecuente es el síndrome de Down, en el que hay una trisomía del cromosoma 21 por la no disyunción en la meiosis en el 95\% de los casos. La incidencia en Cuba es de 7,8 $\times 10.000$ nacidos vivos; en Japón, de $4 \times 10.000$, y en Estados Unidos, de $10,4 \times 10.000$, y es más frecuente en edades maternas avanzadas 5 .

Otra causa de DI es el consumo de alcohol durante el embarazo, que produce el síndrome fetal alcohólico. El alcohol o sus derivados acetilaldehídos interfieren en los procesos metabólicos necesarios para el desarrollo del encéfalo durante la embriogénesis. Ocurre entre el 30 y el 50 de las madres que con- sumen alcohol durante el embarazo ${ }^{6}$. Condiciones perinatales como las distocias del parto, el parto pretérmino, fetos menores de 32 semanas y peso menor de $1.500 \mathrm{~g}$, y la hipoxia neonatal pueden producir daño cerebral y provocar DI. Los antecedentes de DI en familiares de primer grado son elementos importantes para sugerir una herencia multifactorial cuando se excluyen otras causas conocidas.

La literatura consultada afirma que en el 10 al 50\% de los casos de DI no se puede precisar la causa ${ }^{7}$. Pitetti et a ${ }^{8}$ señalan en estudios realizados con dinamometría isocinética que los discapacitados intelectuales presentan menores valores de fuerza muscular en todos los grupos de edades que la población sana. Horvat, al comparar la fuerza muscular de individuos con síndrome de Down y otros con DI de otra etiología, encontró resultados significativamente menores en los primeros.

El propósito de nuestro estudio fue determinar la relación existente entre el desempeńo deportivo y la etiología y el grado de discapacidad, y para ello estudiamos 30 atletas que participaron en los Juegos Nacionales de Olimpiadas Especiales en levantamiento de pesas. Los resultados obtenidos muestran que los atletas con DI ligera alcanzaron mejores resultados en las tres modalidades en que se compitió que los que presentaban un mayor grado de discapacidad, y los atletas con síndrome de Down alcanzaron menores resultados que otros atletas con igual gravedad de discapacidad.

\section{MATERIAL Y MÉTODO}

Se estudiaron 30 atletas masculinos con discapacidad intelectual que participaron en los Juegos Nacionales de Olimpiadas Especiales en levantamiento de pesas, sin limitación físicomotora, a los que se les determinaron las siguientes variables para su clasificación.

- Edad cronológica. Se recogió la fecha de nacimiento por carnet de identificación.

- Peso. Determinado con una balanza de contrapeso DETECTO y con el atleta vestido sólo con ropa interior.

- Etiología. Entrevista con tutor y datos recogidos de la historia clínica.

- Grado de discapacidad. Datos recogidos de la historia clínica.

Para el desempeńo deportivo se aplicó la fórmula total de Schwartz, que consiste en multiplicar el peso levantado por un coeficiente determinado para su peso corporal (regla 1-10 a 13 de la Federación Internacional de Halterofilia [IPF]) en cada una de las modalidades que se compitió. 


\section{Modalidades}

- Press de banca. El atleta debe colocarse extendido sobre banco con la cabeza, el tronco y los glúteos pegados al banco y los pies planos sobre el suelo; debe mantener esta posición mientras ejecute el levantamiento (regla D 1-9 IPF).

- Levantamiento directo. Levantamiento de peso muerto. La barra debe colocarse horizontalmente ante los pies del atleta, que la agarrará con una presa optativa con ambas manos y la levantará con un movimiento continuo hasta ponerla vertical (regla D 1-2 de IPF).

- Squat o flexión de piernas. El atleta debe colocarse con el tronco derecho, la parte superior de la barra transversal en posición horizontal respecto a los hombros, las manos agarrando la barra y los pies planos sobre la plataforma, y entonces flexionar las piernas hasta formar un ángulo no menor de 90 grados (regla D 1-6 IPF).

\section{Análisis estadístico}

Los datos obtenidos de cada grupo se presentan como promedios, medias y desviaciones estándar. Para la comparación se utilizó el test de Mann-Whitney. Las diferencias fueron consideradas estadísticamente significativas para valores de $\mathrm{p}<0,05$.

Se obtuvo el consentimiento informado para la realización de este estudio de todos los atletas.

\section{Discusión}

En nuestra investigación, la etiología más frecuente de DI fue el síndrome de Down, con 7 atletas (23\%), seguida por las distocias de parto, con 5 atletas (16,5\%), sólo superados por las causas desconocidas, con 9 atletas (30\%) (tabla I). Estos porcentajes coinciden con la literatura revisada, que los sitúa entre el 10 y el $50 \%{ }^{9,10}$.

En cuanto al grado de discapacidad, predominó el grado ligero de DI, con 19 atletas (63,3\%). El grado moderado se observó en 7 atletas con síndrome de Down y en 4 cuya etiología no pudo ser definida. La edad y el peso corporal de los grupos estudiados no presentaron diferencias significativas (tablas II y III).

Se observó que los atletas con DI moderada obtuvieron resultados deportivos significativamente menores que los atletas con DI ligera en las tres modalidades en que se compitió: levantamiento de banco, peso muerto y acuclillado (tabla IV), lo que quizá pueda explicarse por la presencia de 7 atletas con síndrome de Down que constituían parte de ese grupo.
Tabla I Etiología de la discapacidad intelectual

\begin{tabular}{|llcc|}
\hline Causas & Entidad & Casos (n) & $\%$ \\
\hline Genéticas $(n=7)$ & Síndrome de Down & 7 & 23,33 \\
\hline $\begin{array}{l}\text { Prenatal ambiental } \\
(n=4)\end{array}$ & Infección materna & I & 3,33 \\
\hline & $\begin{array}{l}\text { Síndrome alcohólico } \\
\text { fetal }\end{array}$ & 3 & 10 \\
\hline Perinatales $(n=7)$ & Distocia de parto & 5 & 16,66 \\
\hline & Parto pretérmino & I & 3,33 \\
\hline Hipoxia neonatal & I & 3,33 \\
\hline Multifactoriales $(n=3)$ & Epilepsia & I & 3,33 \\
\hline & Antecedentes patológicos & 2 & 6,66 \\
\hline familiares de DI & Desconocida & 9 & 30 \\
\hline
\end{tabular}

Tabla II

Discapacidad ligera: edad y peso

\begin{tabular}{|lccc|}
\hline Causa & $\%$ & Edad (años) & Peso $(\mathbf{k g})$ \\
\hline Desconocida $(n=5)$ & 26,3 & $21,8 \pm 3,6$ & $77,2 \pm 9,37$ \\
\hline Distocia de parto $(n=5)$ & 26,3 & $22,8 \pm 3,27$ & $77,48 \pm 24,69$ \\
\hline $\begin{array}{l}\text { Síndrome alcohólico } \\
\text { fetal }(n=3)\end{array}$ & 15,7 & $19 \pm 2,82$ & $77,2 \pm 32,8$ \\
\hline $\begin{array}{l}\text { Antecedentes patológicos } \\
\text { familiares de DI }(n=2)\end{array}$ & 10,5 & 17 & $63,7 \pm 0,42$ \\
\hline Infección materna $(n=1)$ & 5,2 & 19 & 62,1 \\
\hline Parto pretérmino $(n=1)$ & 5,2 & 19 & 61,9 \\
\hline Epilepsia $(n=1)$ & 5,2 & 25 & 71,6 \\
\hline Hipoxia neonatal $(n=1)$ & 5,2 & 26 & 117,7 \\
\hline
\end{tabular}

Tabla III Discapacidad moderada: edad y peso

\begin{tabular}{|lccc|}
\hline Causa & $\%$ & Edad (años) & Peso $(\mathbf{k g})$ \\
\hline Síndrome de Down $(n=7)$ & 23,33 & $26,4 \pm 9,4$ & $64,8 \pm 6,6$ \\
\hline Desconocida $(n=4)$ & 13,33 & $21 \pm 6,7$ & $78,5 \pm 5,4$ \\
\hline
\end{tabular}


TRABAJOS ORIGINALES

Tabla IV

Grado de discapacidad y rendimiento deportivo

\begin{tabular}{|lccc|}
\hline Grado de discapacidad & Levantamiento en banco $^{\mathbf{a}}$ & Peso muerto $^{\mathbf{b}}$ & Acuclillado $^{\mathbf{c}}$ \\
\hline Ligera $(\mathrm{n}=19)$ & $91,0 \pm 22,3$ & $150,0 \pm 29,2$ & $120 \pm 24,3$ \\
\hline Moderada $(\mathrm{n}=1 \mathrm{I})$ & $55,0 \pm 23,5$ & $85,0 \pm 32,7$ & $70,0 \pm 24,2$ \\
\hline
\end{tabular}

${ }^{\mathrm{a}} \mathrm{p}=0,002$.

${ }^{\mathrm{b}} \mathrm{p}=0,007$

${ }^{c} p=0,002$.

Tabla V Discapacidad moderada y rendimiento deportivo

\begin{tabular}{|lcccc|}
\hline Etiología & Peso $(\mathbf{k g})$ & Levantamiento en banco & Peso muerto $^{\mathbf{a}}$ & Acuclillado $^{\mathbf{a}}$ \\
\hline Síndrome de Down $(\mathrm{n}=7)$ & 64,8 & $55,0 \pm 23,56$ & $85,0 \pm 32,7$ & $70,0 \pm 24,22$ \\
\hline Desconocida $(\mathrm{n}=4)$ & 78,5 & $95,2 \pm 8,26$ & $147,7 \pm 26,77$ & $117,2 \pm 37,8 \mathrm{I}$ \\
\hline
\end{tabular}

${ }^{a} \mathrm{p}<0,05$.

Pitetti et $\mathrm{al}^{8,12}$ señalan en estudios realizados con dinamometría isocinética que los discapacitados con síndrome de Down presentan valores bajos de fuerza muscular en los brazos y las piernas.

Cuando comparamos el desempeño deportivo de los atletas con síndrome de Down con otros con igual grado de discapacidad pero de diferente etiología, los primeros mostraron menores valores en su desempeńo $(p<0,05)$ en peso muerto y acuclillado (tabla V). Estos resultados coinciden con estudios realizados por otros autores ${ }^{12,13}$.

Horvat et $\mathrm{al}^{12}$, al comparar la fuerza muscular de individuos con síndrome de Down y otras discapacidades causadas por otra etiología, encontraron resultados significativamente menores en los primeros.

Estudios realizados con dinamometría isocinética para determinar la fuerza muscular de los pacientes con síndrome de Down mostraron valores significativamente menores durante la abducción de cadera y la extensión de la articulación de la rodilla, que el grupo control ${ }^{14}$.

La fuerza muscular en el cuádriceps y bíceps femoral fue significativamente menor en jóvenes atletas con discapacidad intelectual que en el grupo control, en diferentes ángulos medidos con dinamometría isocinética (Cybex II) ${ }^{15}$.

En un estudio comparativo ${ }^{16}$ de sujetos con síndrome de Down, personas con discapacidad intelectual sin síndrome de Down y sujetos sedentarios, al medir el pico de torque mediante dinamometría isocinética a velocidades angulares de 60 y 90 grados/s con Cybex 340, en la flexoentensión del codo se encontraron valores más bajos en el primer grupo; la diferencia entre los sujetos con síndrome de Down y los sujetos sedentarios es significativa.

Debemos señalar que los pacientes con síndrome de Down pueden ser más sensibles a la fatiga, debido al estrés oxidativo que provoca el ejercicio y el ámbito competitivo, ya que las células trisómicas poseen una sobreexpresión de la enzima superóxido dismutasa por encontrarse su gen $S O D-1$ en el cromosoma 21 q22.1, lo que altera el metabolismo de las especies reactivas de oxígeno, con la posterior formación de radicales hidroxilos, considerados el oxidante natural más potente ${ }^{17,18}$.

\section{CONCLUSIONES}

Numerosos estudios coinciden en que la fuerza muscular en sujetos con síndrome de Down es menor que en sujetos con discapacidad intelectual de otras causas. Consideramos que la promoción de la actividad física en esta población constituye un sistema de apoyo para reducir los factores de riesgo asociados al sedentarismo, pues eleva la capacidad cardiorrespiratoria y la fuerza muscular, y proporciona un mayor grado de capacitación. 


\section{Bibliografía}

1. Halsband U, Lange R. Motor learning in man: a review of functional and clinical studies. J Physiol (Paris). 2006;99:414-24.

2. Esparza DY, Larue J. Interacciones cognitivo-motoras: el papel de la representación motora. Rev Neurol. 2008;46:219-24.

3. Goodale MA. Different spaces and different times for perception and action. Prog Brain Res. 2001;134:313-31.

4. Luckasson R, Borthwick-Duffy S, Buatix WH, et al. Mental retardation. Definition, classification and system of support. 10th ed. Washintong, DC: American Association on Mental Retardation, 2002.

5. Revenga Bodi LR, Byo MI, Rosalens MM. Retraso mental de origen genético. Rev Neurol. 2006;43:181.

6. Clarren SK, Ron dal SP, Sanderson M, et al. Screening for fetal alcohol syndrome in primary schools: a feasibility study. Teratology. 2001;63:3-10.

7. González Alvaredo S, Sanz Rojo R, García Santiago J, Gaztañaga Expósito R, Bengoa A, Pérez-Yarza EG. Genetic diagnostic criteria in cases of mental retardation and development of idiopathic origin. An Pediatr (Barc). 2008;69:446-53.

8. Pitetti KH, Climstein M, Mays MJ, Barret PJ. Isokinetic arm and leg strength of adults with Down syndrome: a comparative study. Arch Phys Med Rehabil. 1992;73:847-50.

9. Committee on Genetics. Health supervision for children with Down syndrome. Pediatrics. 2001;107:442-49.

10. Hook EB, Crossp K, Schreinemachers DM. Chromosomal abnormality rates in amniocentesis and live born infants. JAMA. 1983;249:2034-38
11. Balic MG. Síndrome de Down y respuesta al esfuerzo físico. Tesis doctoral. Barcelona: Departamento de Ciencias Morfológicas; 2000.

12. Croce RV, Pitetti KH, Horvat M, Miller J. Peak torque, average power and hamstring/quadriceps ratios in nondisabled adults and adults with mental retardation. Arch Phys Med Rehabil. 1996;77: 369-72.

13. Banyard T, Pitteti KH, Guerra M, Femhall B. Heart rate variability at rest and exercise in person with Down Syndrome. Arch Phys Med Rehabil. 2003;84:1604-8.

14. Mercer VS, Lewis C. Hip abductor and knee extensor muscle strength of children with and without Down Syndrome. Pediatr Phys Therm. 2001;13:18-26.

15. Angelopolaulo N, Matziari C, Tsimaras V, Sakadamis A, Souftas $\mathrm{V}$, Mandroukas K. Bone mineral density and muscle strength in young men with mental retardation (with and without Down Syndrome). Calcif Tissue Int. 2000;66:176-80.

16. Horvat M, Pitteti KH, Croce R. Isokinetic torque, average power, and flexion/extension ratios in nondisabled adults and adults with mental retardation. J Orthop Sports Phys Ther. 1997;25:395-9.

17. Ordoñes FJ, Rosety-Rodriguez M, Rosety M. A 12 week physical activity program increases glucose-6-phosphate deshydrogenase activity in Down Syndrome adolescent. Medicin (Buenos Aires). 2005;65:518-20.

18. Gulesserian T, Seidi R, Hardmeier R, Cairns N, Lubec G. Superoxide dismutase SOD1, encode on chromosome 21, but not SOD2 is overexpressed in brains of patients with Down Syndrome. J Invest Med. 2001;49:41-6. 